\title{
How to Optimize Trichoscopy for Evaluation of Scalp Vessels
}

\author{
Agata Kłosowicz ${ }^{\text {a }}$ Waleed Alsalhi ${ }^{\mathrm{b}} \quad$ Antonella Tosti $^{\mathrm{c}}$ \\ aDepartment of Dermatology, University Hospital in Kraków, Kraków, Poland; 'bepartment of Dermatology, \\ College of Medicine, Majmaah University, Al-Majmaah, Saudi Arabia; ' Dr. Phillip Frost Department of Dermatology \\ and Cutaneous Surgery, University of Miami Miller School of Medicine, Miami, FL, USA
}

\section{Keywords}

Hair loss · Trichoscopy - Scalp psoriasis - Scalp vessels .

Seborrheic dermatitis

\begin{abstract}
Introduction: Trichoscopy greatly facilitates clinical diagnosis in patients with hair loss and may decrease the necessity for histopathological examination. Structures which may be revealed by trichoscopy include hair shafts, hair follicle openings, the perifollicular epidermis, and cutaneous microvessels. Cutaneous microvessels revealed in trichoscopy may vary in type and number depending on the scalp area, type of the disease, and its activity. Firm direct pressure (diascopy) might result in their blanching, but as of yet, there are no studies on the types of vessels that do or do not blanch on applying pressure. Methods: We studied interfollicular twisted loops in 16 cases of biopsy-confirmed scalp psoriasis and 37 cases of arborizing vessels in normal subjects and seborrheic dermatitis patients. Results: We observed that all arborizing red line vessels blanched on applying pressure. Instead, the vast majority of twisted and simple loops, despite their vascular nature, did not blanch on performing diascopy. Conclusion: In the light of these findings, diascopy
\end{abstract}

of vascular changes in hair loss patients might provide additional clues for a proper diagnosis, especially in differentiating scalp psoriasis, seborrheic dermatitis, and discoid lupus erythematosus.

(c) 2020 S. Karger AG, Basel

\section{Introduction}

Trichoscopy greatly facilitates the clinical diagnosis in patients with hair loss and may decrease the necessity for histopathological examination. Structures which may be revealed by trichoscopy include hair shafts, hair follicle openings, the perifollicular epidermis, and cutaneous microvessels [1].

Evaluation of the vascular structures depends on the choice of optical devices and dermoscopic imaging techniques [2]. To visualize distinctive morphological features of the cutaneous vasculature on the scalp, it is important to perform videodermoscopy with the epiluminescent mode of operation. Firm direct pressure (diascopy) might result in their blanching, but as of yet, there are no studies on the types of vessels that do or do not blanch on applying pressure.

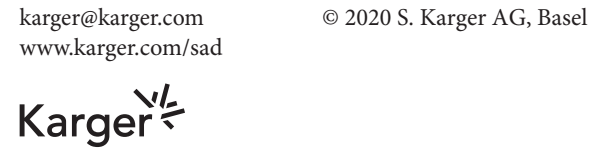



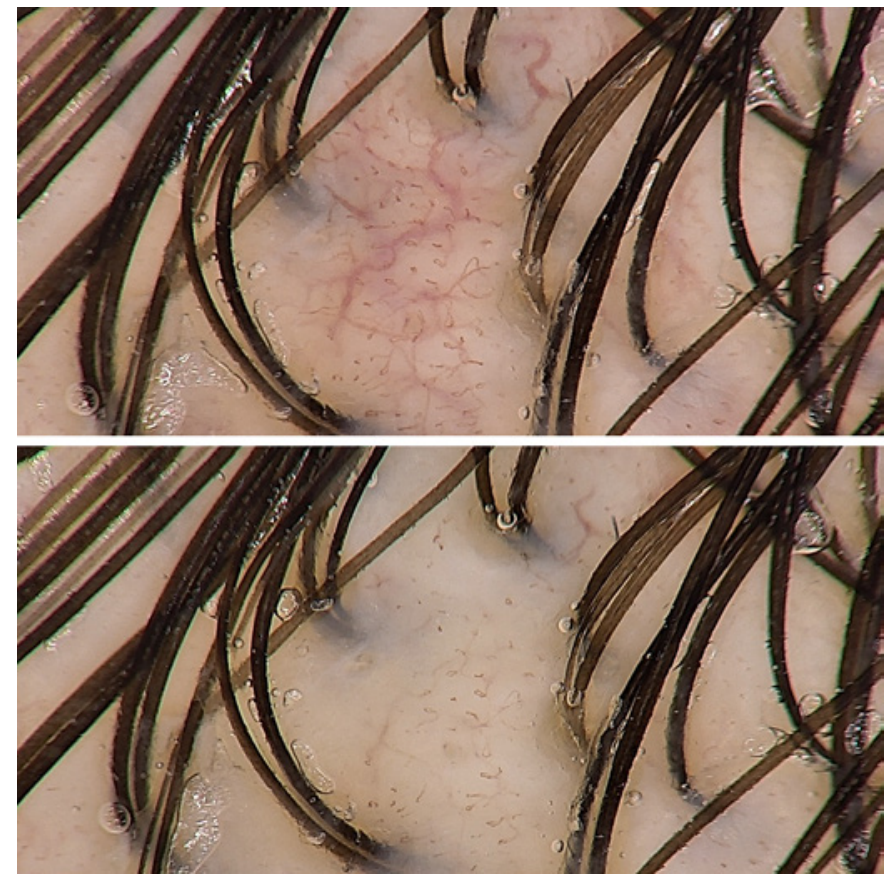

Fig. 1. Arborizing vessels in seborrheic dermatitis without (top) and with pressure (bottom).

\section{Material and Methods}

We studied interfollicular twisted loops in 16 cases of biopsyconfirmed scalp psoriasis and 37 cases of arborizing vessels in normal subjects and seborrheic dermatitis patients. We always took the picture without pressure first and then without moving the lens, we applied pressure and documented a second one using the FotoFinder $\times 40$ magnification.

\section{Results}

We observed that all arborizing red line vessels blanched on applying pressure (shown in Fig. 1). Instead, the vast majority of twisted and simple loops (15/16), despite their vascular nature, did not blanch on performing diascopy (shown in Fig. 2).

\section{Discussion}

The skin blood supply originates from perforating vessels rising from the underlying muscles and subcutaneous fat to form a plexus, the lower horizontal plexus, at the dermal-subcutaneous interface. From the lower plexus, paired arterioles and venules rise to form direct connec-
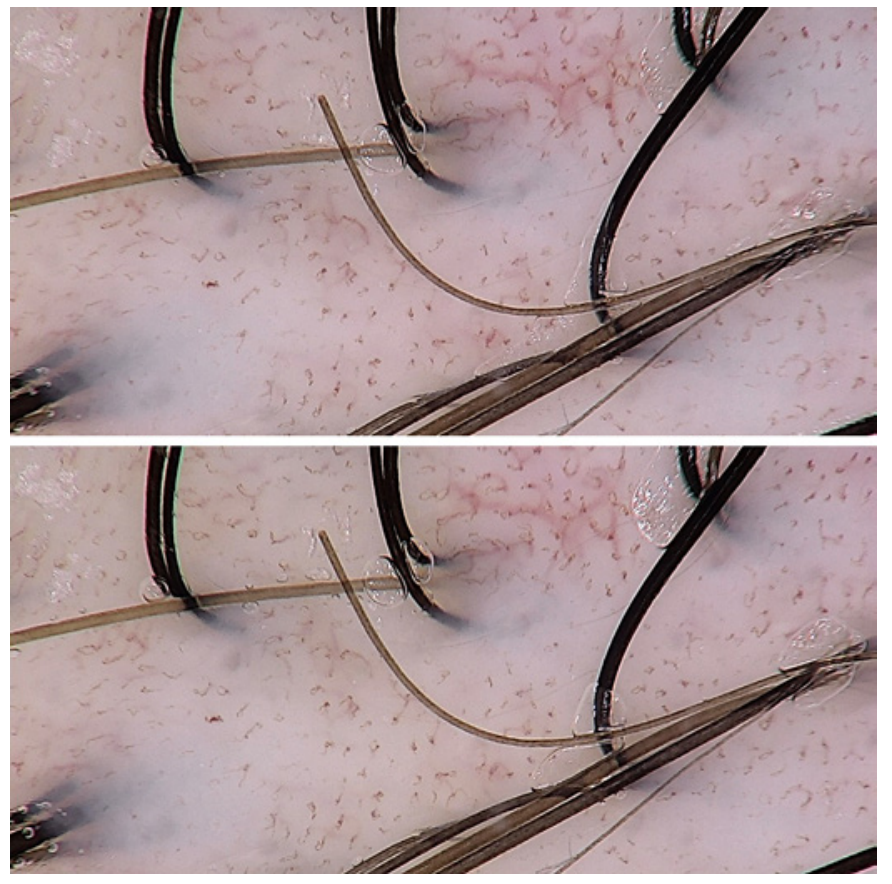

Fig. 2. Interfollicular twisted loops in psoriasis without (top) and with pressure (bottom).

tions with a second plexus in the papillary dermis (the subpapillary plexus), and from this, the capillary loops of the dermal papillae arise. The majority of the microvasculature of the skin resides in the papillary dermis 1-2 mm below the surface of the skin [3].

Cutaneous microvessels revealed in trichoscopy may vary in type and number depending on the scalp area, type of the disease, and its activity. Various vascular patterns in healthy individuals and in patients with specific scalp disorders have been best described in the literature $[2,4,5]$. Three major characteristic patterns described by Tosti and Duque-Estrada $[6,7]$ include the following.

\section{Interfollicular Simple Red Loops}

Interfollicular simple red loops are seen in healthy individuals and may be present in the majority of inflammatory conditions. They present as regularly spaced multiple fine, red, hairpin-shaped structures. Their distribution can be patchy or diffuse. They are best seen at higher magnifications $(x 50$ or higher $)$ with the camera probe angled tangentially. At lower magnifications, they present as pinpoint red dots. In healthy females, pinpoint-like vessels are commonly seen in frontal scalp [5]. Histopathologically, they correspond to capillaries in the dermal papillae. Absent loops may indicate epidermal atrophy. 


\section{Interfollicular Twisted Loops}

These tortuous and dilated blood vessels appear as twisted coils that are best visualized with the probe placed tangentially to the scalp surface. Twisted loops are present in some of the conditions characterized by acanthosis, such as psoriasis and folliculitis decalvans. Apart from the polytrichia, which is a trichoscopic hallmark of folliculitis decalvans [8], the presence of interfollicular twisted red loops localized around affected follicles is one of the most common findings for this type of scarring alopecia [9]. According to Kim et al. [10], twisted red loops can help differentiate scalp psoriasis from seborrheic dermatitis as this trichoscopic feature was observed in 29 patients (53\%) with psoriasis and only 9 patients (22\%) with seborrheic dermatitis. These data are not consistent with the results of a study by Kibar et al. [11], in which red twisted loops were more often seen in seborrheic dermatitis than in scalp psoriasis (55 vs. $48 \%$ ).

\section{Arborizing Red Lines}

These tree branch-like vessels of larger caliber are seen as lines that underlie and articulate to the loops in normal and affected scalp. These are best visualized at higher magnifications with tangential viewing. They correspond to the ectatic subpapillary vascular plexus. In healthy individuals, arborizing red lines focally dominate in occipital and temporal areas [5]. Arborizing vessels constitute a pattern commonly seen in cicatricial alopecia caused by discoid lupus erythematosus. Thin arborizing vessels radially emerging from large yellow dots are considered a characteristic feature for DLE, in the literature referred as "red spider in yellow dot" [12]. The visualization of arborizing red lines may facilitate the diagnosis of seborrheic dermatosis and help differentiate this condition from scalp psoriasis. The incidence of arborizing vessels in seborrheic dermatitis patients varied from 49 percent to 73 percent, depending on the study and was significantly higher in comparison to scalp psoriasis patients (9-35\%) $[10,11]$. performed to determine whether the lesion is vascular or hemorrhagic. Diascopy is based on the principle that pressing a glass slide against a vascular skin lesion results in its blanching as the blood dissipates intravascularly, and the tissue acquires a blanched appearance. Hemorrhagic lesions, such as petechiae or purpura do not blanch on applying pressure due to the presence of extravasated blood in the tissues [13]. sels which blanch on applying pressure (Fig. 1), twisted

and simple loops, despite their vascular nature, do not blanch on performing diascopy (Fig. 2). We hypothesize that this phenomenon is due to the fact that arborizing red lines run horizontally, parallel to the skin, while twisted and simple loops are oriented perpendicularly to the surface. In the light of these findings, diascopy of vascular changes in hair loss patients might provide additional clues for a proper diagnosis, especially in differentiating scalp psoriasis, seborrheic dermatitis, and discoid lupus erythematosus.

\section{Statement of Ethics}

Subjects gave their written informed consent. Ethics approval was not required.

\section{Disclosure Statement}

The authors have no conflicts of interest to disclose.

\section{Funding Sources}

The authors did not receive any funding.

\section{Author Contributions}

Article conception and design: A. Tosti, A. Kłosowicz, and W. Alsalhi. Acquisition of data: A. Tosti, W. Alsalhi, and A. Kłosowicz. Analysis and interpretation: A. Kłosowicz, A. Tosti, and W. Alsalhi. Preparation of the outline of the manuscript: A. Kłosowicz, A. Tosti, and W. Alsalhi. Literature review: A. Kłosowicz and A. Tosti. Critical review and supervision: A. Tosti and W. Alsalhi.

Diascopy is considered a simple test for blanchability

We observed that in contrast to arborizing red line ves-

\section{References}

1 Ross EK, Vincenzi C, Tosti A. Videodermoscopy in the evaluation of hair and scalp disorders. J Am Acad Dermatol. 2006;55(5):799806

2 Wang SQ, Dusza SW, Scope A, Braun RP, Kopf AW, Marghoob AA. Differences in dermoscopic images from nonpolarized dermoscope and polarized dermoscope influence the diagnostic accuracy and confidence level: a pilot study. Dermatol Surg. 2008;34(10): 1389-95.

3 Shore AC. Capillaroscopy and the measurement of capillary pressure. Br J Clin Pharmacol. 2000;50(6):501-13.

4 Rudnicka L, Olszewska M, Rakowska A, Slowinska M. Trichoscopy update 2011. J Dermatol Case Rep. 2011;5(4):82-8. 
5 Rakowska A. Trichoscopy (hair and scalp videodermoscopy) in the healthy female. Method standardization and norms for measurable parameters. J Dermatol Case Rep. 2009;3(1): 14-9.

6 Tosti A. Hair shaft disorders. In: Tosti A, editor. Dermoscopy of hair and scalp: pathological and clinical correlation. Illustrated ed. USA: CRC Press; 2007. p. 51-3.

7 Tosti A, Duque-Estrada B. Dermoscopy in hair disorders. J Egypt Womens Dermatol Soc. 2010;7:1-4.
8 Vañó-Galván S, Molina-Ruiz AM, Fernández-Crehuet P, Rodrigues-Barata AR, AriasSantiago S, Serrano-Falcón C, et al. Folliculitis decalvans: a multicentre review of $82 \mathrm{pa}$ tients. J Eur Acad Dermatol Venereol. 2015; 29:1750-7.

9 Duque-Estrada B, Estrada BD, Tamler C, Sodré CT, Barcaui CB, Pereira FB. Dermoscopy patterns of cicatricial alopecia resulting from discoid lupus erythematosus and lichen planopilaris. An Bras Dermatol. 2010;85(2):17983.

10 Kim GW, Jung HJ, Ko HC, Kim MB, Lee WJ, Lee SJ, et al. Dermoscopy can be useful in differentiating scalp psoriasis from seborrhoeic dermatitis. Br J Dermatol. 2011;164(3):652-6.
11 Kibar M, Aktan Ş, Bilgin M. Dermoscopic findings in scalp psoriasis and seborrheic dermatitis; two new signs; signet ring vessel and hidden hair. Indian J Dermatol. 2015;60(1): 41-5.

12 Rakowska A, Slowinska M, Kowalska-Oledzka E, Warszawik O, Czuwara J, Olszewska M, et al. Trichoscopy of cicatricial alopecia 2011. J Drugs Dermatol. 2012 Jun;11(6):753-8.

13 Rudd M, Eversole R, Carpenter W. Diascopy: a clinical technique for the diagnosis of vascular lesions. Gen Dent. 2001;49(2):206-9. 